\title{
Geometria de Laguerre e hipersuperfícies de Dupin com curvaturas de Laguerre constantes em $\mathbb{R}^{n+1} \mid 1$
}

\author{
Laguerre geometry and Dupin hypersurfaces with constant Laguerre \\ curvatures in $\mathbb{R}^{n+1}$
}

\author{
Fernanda Alves Caixeta \\ Luciana Maria Dias de Ávila Rodrigueș
}

Resumo. Neste trabalho, apresentamos os resultados estudados em Caixeta e Rodrigues [4]. Inicialmente, estudamos a geometria das esferas orientadas em $\mathbb{R}^{n+1}$ tendo como base o trabalho de Cecil [1], e a geometria de Laguerre no espaço Euclidiano, de acordo com o artigo de Li e Wang [6]. Posteriormente, considerando $M^{n} \subset \mathbb{R}^{n+1}$ uma hipersuperfície orientável com $r$ curvaturas principais distintas $(r \geq 3)$ e não nulas, apresentamos uma caracterização obtida por Li e Wang [7], em termos dos invariantes de Laguerre, das hipersuperfícies de Dupin com curvaturas de Laguerre constantes. Apresentamos ainda o resultado de classificação das hipersuperfícies de Dupin com curvaturas de Laguerre constantes proposto por Li e Wang [7], que consiste em mostrar que uma hipersuperfície de Dupin com curvaturas de Laguerre constantes é Laguerre equivalente a uma hipersuperfície isoparamétrica de Laguerre plana. Em um contexto um pouco diferente do até então estudado, considerando uma hipersuperfície de Dupin própria em $\mathbb{R}^{n+1}$, que admite um sistema de coordenadas principais com $n$ curvaturas principais distintas e não nulas, Cezana e Tenenblat [2] apresentaram uma caracterização das hipersuperfícies de Dupin em $\mathbb{R}^{n+1}$, $n \geq 3$, com todas as curvaturas principais distintas e curvaturas de Laguerre constantes, que admite uma parametrização por linhas de curvatura, em termos de seus raios de curvatura e de sua primeira forma fundamental. Assim, utilizando esse resultado, Cezana e Tenenblat [2] apresentaram explicitamente todas essas hipersuperfícies que possuem curvaturas de Laguerre constantes.

Palavras-chave. Geometria de Laguerre. Hipersuperfície de Dupin. Curvaturas de Laguerre. Hipersuperfície isoparamétrica de Laguerre.

\footnotetext{
${ }^{1}$ Trabalho apresentado na X Semana de Matemática do Pontal, 2019.

${ }^{2}$ Departamento de Matemática, Universidade de Brasília, fernandacaixeta@outlook.com

${ }^{3}$ Departamento de Matemática, Universidade de Brasília, luavila@unb.com
} 


\begin{abstract}
In this work, we present the results studied in Caixeta e Rodrigues [4]. Initially, we studied the geometry of the oriented spheres in $\mathbb{R}^{n+1}$ based on the work of Cecil [1], and the Laguerre geometry in the Euclidean space, according to the article by Li e Wang [6]. Subsequently, considering $M^{n} \subset \mathbb{R}^{n+1}$ an oriented hypersurface with $r(r \geq 3)$ distinct nonvanishing principal curvatures, we present a characterization obtained by Li e Wang [7], in terms of Laguerre invariants, of Dupin hypersurfaces with constant Laguerre curvatures. We also present the classification result of the Dupin hypersurfaces with constant Laguerre curvatures proposed by Li e Wang [7], which consists in showing that a Dupin hypersurfaces with constant Laguerre curvatures is Laguerre equivalent to a flat Laguerre isoparametric hypersurface. In a slightly different context from the one studied so far, considering a proper Dupin hypersurfaces of the Euclidean space $\mathbb{R}^{n+1}$, that admit principal coordinate systems and have $n$ distinct nonvanishing principal curvatures Cezana e Tenenblat [2] presented a characterization of Dupin hypersurfaces in $\mathbb{R}^{n+1}, n \geq 3$, with all the distinct principal curvatures and constant Laguerre curvatures, that is parametrized by lines of curvature, in terms of the radius of curvature and their first fundamental form. So, using this result Cezana e Tenenblat [2] showed explicitly all such hypersurfaces that have constant Laguerre curvatures.
\end{abstract}

Keywords. Laguerre geometry. Dupin hypersurfaces. Laguerre curvatures. Laguerre isoparametric hypersurfaces.

\title{
1 Introdução
}

Uma hipersuperfície $M^{n}$ em uma forma espacial $\mathbb{R}^{n+1}, \mathbb{S}^{n+1}$ ou $\mathbb{H}^{n+1}$ é de Dupin se cada curvatura principal é constante ao longo da correspondente linha de curvatura. Uma hipersuperfície de Dupin $M^{n}$ é dita própria se o número $r$ de curvaturas principais é constante em $M^{n}$. Os primeiros exemplos dessas superfícies, obtidos por Dupin em 1822, foram as cíclides de Dupin. Desde então, as hipersuperfícies de Dupin passaram a ser objeto de estudo para muitos pesquisadores, sobretudo acerca de problemas envolvendo a classificação dessas hipersuperfícies (veja, por exemplo, [5], [7], [8], [9], [11], dentre outros).

Uma importante classe de exemplos de hipersuperfícies de Dupin, são as hipersuperfícies isoparamétricas em $\mathbb{R}^{n+1}, \mathbb{S}^{n+1}$ ou $\mathbb{H}^{n+1}$. Uma hipersuperfície é dita isoparamétrica se ela possui curvaturas principais constantes. Num trabalho publicado em 1999, Corro, Fereira e Tenenblat [3] aplicaram transformações de Ribaucour ao hiperplano, para obter 
uma família de hipersuperfícies de Dupin em $\mathbb{R}^{n+1}, n \geq 3$, parametrizada por linhas de curvatura com $n$ curvaturas principais distintas, dada, a menos de movimentos rígidos por,

$$
x\left(u_{1}, u_{2}, \ldots, u_{n}\right)=\left(u_{1}, u_{2}, \ldots, u_{n}, 0\right)-\frac{\sum_{j=1}^{n} b_{j} u_{j}^{2}+\gamma}{\sum_{j=1}^{n} b_{j}^{2} u_{j}^{2}+1}\left(b_{1} u_{1}, b_{2} u_{2}, \ldots, b_{n} u_{n},-1\right),
$$

em que $b_{j}$ são números reais distintos e não nulos e $\gamma \in \mathbb{R}$. Essa família de hipersuperfícies de Dupin tem curvatura de Lie constante, curvatura de Laguerre constante e curvatura de Möbius não constante. Além disso, é um exemplo de uma família de hipersuperfícies isoparamétricas de Laguerre planas utilizado na classificação das hipersuperfícies de Dupin com curvaturas de Laguerre constantes.

O estudo das hipersuperfícies de Dupin, e de problemas envolvendo a classificação dessas hipersuperfícies, pode ser contextualizado no cenário das esferas de Lie. Em 1985, Pinkall [10] provou, dentre outros resultados, que a propriedade de uma hipersuperfície ser de Dupin própria é invariante pela ação do grupo das transformações de Lie. Assim, a classificação das hipersuperfícies de Dupin é realizada a menos dessas transformações. O grupo das transformações de Lie pode ser visto como a união de dois importantes subgrupos, o subgrupo das transformações de Möbius e o subgrupo das transformações de Laguerre.

Na geometria diferencial de Laguerre, são estudadas as propriedades de hipersuperfícies de Laguerre invariantes pelo grupo das transformações de Laguerre em $U \mathbb{R}^{n+1}$. Em 2007, Li e Wang [6] considerando uma hipersuperfície $x: M^{n} \rightarrow \mathbb{R}^{n+1}$ sem pontos umbílicos com curvaturas principais distintas não nulas, definiram os invariantes básicos de Laguerre, a saber, uma métrica invariante de Laguerre $g$, segunda forma fundamental de Laguerre $\mathbb{B}$, forma de Laguerre $\mathbb{C}$ e o tensor de Laguerre $\mathbb{L}$. Além disso, definiram as formas espaciais de Laguerre $U \mathbb{R}^{n+1}, U \mathbb{R}_{1}^{n+1}$ e $U \mathbb{R}_{0}^{n+1}$ e as imersões de Laguerre $\sigma: U \mathbb{R}_{1}^{n+1} \rightarrow U \mathbb{R}^{n+1}$ e $\tau: U \mathbb{R}_{0}^{n+1} \rightarrow U \mathbb{R}^{n+1}$. Usando estas imersões de Laguerre, mostraram que cada hipersuperfície $x: M^{n} \rightarrow \mathbb{R}_{1}^{n+1}$ ou $x: M^{n} \rightarrow \mathbb{R}_{0}^{n+1}$ corresponde a uma hipersuperfície $x^{\prime}: M^{n} \rightarrow \mathbb{R}^{n+1}$.

Em 2010, Li, Li e Wang [8] introduziram o conceito de hipersuperfície com segunda forma fundamental de Laguerre $\mathbb{B}$ paralela e obtiveram a classificação completa dessas hipersuperfícies. Isto é, mostraram que a menos de transformação de Laguerre, as únicas hipersuperfícies com segunda forma fundamental $\mathbb{B}$ paralela em $\mathbb{R}^{n+1}$ são as cíclides de Dupin ou a imagem pela imersão de Laguerre $\tau$ da hipersuperfície $x: M^{n} \rightarrow \mathbb{R}_{0}^{n+1}$ que corresponde, a menos de uma translação em $\mathbb{R}^{n+1}$, a hipersuperfície descrita em (1) quando $\gamma=0$.

Posteriormente, Li e Sun em [9] definiram hipersuperfícies isoparamétricas de La- 
guerre e apresentaram uma classificação dessas hipersuperfícies no caso $M^{3} \subset \mathbb{R}^{4}$. Mostraram também, que uma hipersuperfície isoparamétrica de Laguerre é uma hipersuperfície de Dupin e reciprocamente, uma hipersuperfície de Dupin, com a hipótese adicional de ter a forma de Laguerre $\mathbb{C}$ nula, é uma hipersuperfície isoparamétrica de Laguerre.

Em 2015, Li e Wang [7], fazendo uso de um resultado de caracterização das hipersuperfícies de Dupin com curvaturas de Laguerre constante, provaram que uma hipersuperfície de Dupin $M^{n} \subset \mathbb{R}^{n+1}$, com $r$ curvaturas principais distintas $(r \geq 3)$ e não nulas, é Laguerre equivalente a uma hipersuperfície isoparamétrica de Laguerre plana em $\mathbb{R}^{n+1}$ se, e somente se, as curvaturas de Laguerre são constantes.

Recentemente, em 2017, Cezana e Tenenblat [2] apresentaram algumas propriedades básicas das hipersuperfícies de Dupin com $n$ curvaturas principais distintas. Em seguida, mostraram que qualquer hipersuperfície de Dupin própria em $\mathbb{R}^{n+1}$ com $n$ curvaturas principais distintas e não nulas, que admite um sistema de coordenadas principais e possui curvaturas de Laguerre constantes é dada pela família descrita em (1).

Neste trabalho, estudamos primeiramente a geometria das esferas orientadas em $\mathbb{R}^{n+1}$, tendo como base o trabalho de Cecil [1], e a geometria de Laguerre em $\mathbb{R}^{n+1}$, de acordo com o artigo de Li e Wang [6]. Posteriormente, estudamos os resultados de classificação das hipersuperfícies de Dupin com curvaturas de Laguerre constantes apresentados por Li e Wang [7] e Cezana e Tenenblat [2].

Nosso objetivo é apresentar o resultado proposto por Li e Wang [7], observando que as hipersuperfícies isoparamétricas de Laguerre planas constituem uma classe contida na família dada por (1) quando $\gamma=0$. Em seguida, descrevemos os resultados obtidos por Cezana e Tenenblat [2].

\section{Geometria de Laguerre no espaço Euclidiano}

A fim de apresentarmos os resultados de classificação obtidos por Li e Wang [7] e Cezana e Tenenblat [2], necessitamos de um estudo preliminar a respeito da geometria das esferas orientadas em $\mathbb{R}^{n+1}$ e da geometria diferencial de Laguerre.

Considere sobre $\mathbb{R}^{n+1}$ o fibrado tangente unitário, definido por:

$$
U \mathbb{R}^{n+1}=\left\{(x, \xi) / x \in \mathbb{R}^{n+1}, \xi \in \mathbb{S}^{n}\right\}=\mathbb{R}^{n+1} \times \mathbb{S}^{n} \subset \mathbb{R}^{2 n+2}
$$

Uma esfera orientada $U \mathbb{R}^{n+1}$ é definida por:

Definição 1. Uma esfera orientada em $U \mathbb{R}^{n+1}$ de centro p e raio $r$ é uma subvariedade 
n-dimensional em $U \mathbb{R}^{n+1}$ dada por:

$$
S(p, r)=\left\{(x, \xi) \in U \mathbb{R}^{n+1} / x-p=r \xi\right\}
$$

Geometricamente, $S(p, r)$ com $r \neq 0$ corresponde a uma esfera orientada em $\mathbb{R}^{n+1}$ de centro $p \in \mathbb{R}^{n+1}$ e raio $r$. Se $r>0$ (respectivamente $r<0$ ), o vetor normal unitário $\xi$ de $S(p, r)$ é exterior (respectivamente interior). Se $r=0$, então $S(p, 0)$ representa todos os vetores tangentes a $p$. Denotamos $S(p, 0)$ o ponto esférico em $p \in \mathbb{R}^{n+1}$.

Definimos um hiperplano orientado em $U \mathbb{R}^{n+1}$ como sendo:

Definição 2. Dado $\xi \in \mathbb{S}^{n}$ e uma constante $\lambda \in \mathbb{R}$, dizemos que um hiperplano orientado em $U \mathbb{R}^{n+1}$ é uma subvariedade n-dimensional em $U \mathbb{R}^{n+1}$ dada por:

$$
P(\xi, \lambda)=\left\{(x, \xi) \in U \mathbb{R}^{n+1} / x \cdot \xi=\lambda\right\}
$$

em que “.” é a métrica Euclidiana de $\mathbb{R}^{n+1}$.

Geometricamente, $P(\xi, \lambda)$ é o hiperplano $\left\{x \in \mathbb{R}^{n+1} / x . \xi=\lambda\right\}$ em $\mathbb{R}^{n+1}$ com normal unitário $\xi$.

Considere o conjunto $\Sigma=\{\Gamma / \Gamma$ é esfera ou $\Gamma$ é hiperplano $\} \subset \mathbb{U R}^{n+1}$.

Consideramos $\mathbb{R}_{2}^{n+4}$ o espaço $\mathbb{R}^{n+4}$ munido com o produto interno

$$
\langle X, Y\rangle=-X_{1} Y_{1}+X_{2} Y_{2}+\cdots+X_{n+3} Y_{n+3}-X_{n+4} Y_{n+4}, \quad \forall X, Y \in \mathbb{R}^{n+4}
$$

Definimos a quádrica de Lie como sendo:

Definição 3. No espaço projetivo real $\mathbb{P}^{n+3}$, definimos a quádrica $\mathbb{Q}^{n+2}$ por

$$
\mathbb{Q}^{n+2}=\left\{[X] \in \mathbb{P}^{n+3} /\langle X, X\rangle=0\right\} .
$$

$\mathbb{Q}^{n+2}$ é denotada quádrica de Lie.

Descrevemos uma correspondência bijetiva entre os elementos do conjunto $\Sigma$ e os pontos da quádrica de Lie $\mathbb{Q}^{n+2}$ como segue:

\begin{tabular}{|c|c|}
\hline$\Sigma$ & $\mathbb{Q}^{n+2}$ \\
\hline$S(p, r)$ & {$\left[\left(\frac{1+p \cdot p-r^{2}}{2}, \frac{1-p \cdot p+r^{2}}{2}, p, r\right)\right]$} \\
\hline$P(\xi, \lambda)$ & {$[(\lambda,-\lambda, \xi, 1)]$} \\
\hline$u \in \mathbb{R}^{n+1}$ & {$\left[\left(\frac{1+u \cdot u}{2}, \frac{1-u \cdot u}{2}, u, 0\right)\right]$} \\
\hline
\end{tabular}

Chamamos $[\gamma] \in \mathbb{Q}^{n+2}$ de coordenadas esféricas de $S(p, r)$ e $P(\xi, \lambda)$. 
A correspondência $\Gamma \in \Sigma \longleftrightarrow[\gamma] \in \mathbb{Q}^{n+2}$ define uma bijeção de $\Sigma$ em $\mathbb{Q}^{n+2}-\{[P]\}$, onde

$$
P=(1,-\overrightarrow{1}, \overrightarrow{0}, 0), \overrightarrow{0} \in \mathbb{R}^{n+1}
$$

Com isso, fica determinado um difeomorfismo $L$ entre $U \mathbb{R}^{n+1}$ e o conjunto de todas as retas projetivas contidas em $\mathbb{Q}^{n+2}-\{[P]\}$, chamado difeomorfismo de Lie. Além disso, definimos o grupo das transformações de Laguerre $L \mathbb{G}$ em $U \mathbb{R}^{n+1}$, que é um importante subgrupo do grupo das transformações de Lie, como sendo

$$
L \mathbb{G}=\{T \in \mathbb{O}(n+2,2) / P T=P\},
$$

onde $P=(1,-1, \overrightarrow{0}, 0), \overrightarrow{0} \in \mathbb{R}^{n+1}$ é o vetor tipo luz em $\mathbb{R}_{2}^{n+4}$ e $\mathbb{O}(n+2,2)$ é formado pelo grupo ortogonal que deixa o produto interno (5) invariante.

Considerando $T \in L \mathbb{G}$ então $T$ induz uma transformação projetiva em $\mathbb{P}^{n+3}$, que pode ser restrita a $\mathbb{Q}^{n+2}$, dada por

$$
T([X])=[X T], X \in \mathbb{Q}^{n+2}
$$

Chamamos, $T \in L \mathbb{G}$ e $T: \mathbb{Q}^{n+2} \longrightarrow \mathbb{Q}^{n+2}$, de transformações de Laguerre e $L \mathbb{G}$ o grupo das transformações de Laguerre. Considerando o difeomorfismo de Lie $L$, qualquer $T \in$ $L \mathbb{G}$ induz uma transformação

$$
\phi=L^{-1} \circ T \circ L: U \mathbb{R}^{n+1} \longrightarrow U \mathbb{R}^{n+1},
$$

chamada de transformação de Laguerre em $U \mathbb{R}^{n+1}$, em que o grupo das transformações de Laguerre sobre $U \mathbb{R}^{n+1}$ é dado por

$$
L \mathbb{G}=\left\{\phi: U \mathbb{R}^{n+1} \longrightarrow U \mathbb{R}^{n+1} / \phi=L^{-1} \circ T \circ L, T \in \mathbb{O}(n+2,2), P T=P\right\} .
$$

Assim, definimos uma hipersuperfície de Laguerre em $U \mathbb{R}^{n+1}$ como sendo:

Definição 4. Seja $x: M^{n} \longrightarrow \mathbb{R}^{n+1}$ uma hipersuperfície orientada com curvaturas principais que não se anulam e $\xi: M^{n} \longrightarrow \mathbb{S}^{n}$ a aplicação normal. Uma hipersuperfície $f=(x, \xi): M^{n} \longrightarrow U \mathbb{R}^{n+1}$ é uma hipersuperfície de Laguerre, se $\xi: M^{n} \longrightarrow \mathbb{S}^{n}$ é imersão e se $d x \cdot \xi=0$.

No que segue, apresentamos o conceito de hipersuperfícies serem Laguerre equivalentes.

Definição 5. Sejam $x, \tilde{x}: M^{n} \longrightarrow \mathbb{R}^{n+1}$ duas hipersuperfícies orientadas com curvaturas principais que não se anulam. Dizemos que $x$ e $\tilde{x}$ são equivalentes por transformações de Laguerre (Laguerre equivalentes), se as hipersuperfícies de Laguerre correspondentes 
$f=(x, \xi), \tilde{f}=(\tilde{x}, \tilde{\xi}): M^{n} \longrightarrow U \mathbb{R}^{n+1}$ são diferentes apenas por uma transformação de Laguerre, isto é, $\tilde{f}=\phi \circ f$, onde $\phi=L^{-1} \circ T \circ L, T \in L \mathbb{G}$.

Na geometria diferencial de Laguerre são estudadas as propriedades das hipersuperfícies de Laguerre em $U \mathbb{R}^{n+1}$ que são invariantes pelo grupo das transformações de Laguerre em $U \mathbb{R}^{n+1}$. Objetos invariantes por transformações de Laguerre são chamados por invariantes de Laguerre.

No que segue, apresentamos alguns invariantes básicos de Laguerre para hipersuperfícies de Laguerre em $\mathbb{R}^{n+1}$, estudados pelos autores Li e Wang [6], tais como, a segunda forma fundamental de Laguerre $\mathbb{B}$, a forma de Laguerre $\mathbb{C}$ e o tensor de Laguerre $\mathbb{L}$.

Sejam $x: M^{n} \longrightarrow \mathbb{R}^{n+1}$ uma hipersuperfície orientável sem pontos umbílicos e com curvaturas principais que não se anulam $k_{i}$ e $\xi: M^{n} \rightarrow \mathbb{S}^{n}$ o campo normal unitário. Sejam

$$
r_{i}=\frac{1}{k_{i}} \text { e } r=\frac{r_{1}+\cdots+r_{n}}{n}
$$

o raio de curvatura e o raio de curvatura média de $x$. Definimos a aplicação

$$
Y=\rho(x \cdot \xi,-x \cdot \xi, \xi, 1): M^{n} \longrightarrow C^{n+3} \subset \mathbb{R}_{2}^{n+4},
$$

onde $\rho=\sqrt{\sum_{i}\left(r-r_{i}\right)^{2}}$ e $C^{n+3}$ é o cone de luz definido por

$$
C^{n+3}=\left\{x \in \mathbb{R}_{2}^{n+4} /\langle x, x\rangle=0\right\}
$$

O vetor $Y$ é denotado o vetor posição de Laguerre da imersão $x$.

O teorema seguinte, apresentado por Li e Wang [6], fornece uma equivalência entre hipersuperfícies de Laguerre em termos dos seus respectivos vetores posição.

Teorema 1. Sejam $x, \tilde{x}: M^{n} \longrightarrow \mathbb{R}^{n+1}$ duas hipersuperfícies sem pontos umbílicos $e$ com curvaturas principais que não se anulam. Então $x$ e $\tilde{x}$ são equivalentes por uma transformação de Laguerre se, e somente se, existe $T \in L \mathbb{G}$ tal que $\tilde{Y}=Y T$.

Os autores Li e Wang [6], com base no Teorema 1, mostraram que

$$
g=\langle d Y, d Y\rangle=\rho^{2} d \xi . d \xi
$$

é uma métrica invariante por transformações de Laguerre, chamada a métrica de Laguerre de $x$. 
Seja $\Delta$ o operador Laplaciano de $g$, definimos

$$
\begin{aligned}
N & =\frac{1}{n} \Delta Y+\frac{1}{2 n^{2}}\langle\Delta Y, \Delta Y\rangle Y, \\
\eta & =\left(\frac{1}{2}\left(1+|x|^{2}\right), \frac{1}{2}\left(1-|x|^{2}\right), x, 0\right)+r(x \cdot \xi,-x \cdot \xi, \xi, 1),
\end{aligned}
$$

onde $r=\frac{r_{1}+\ldots+r_{n}}{n}$ é o raio de curvatura média de $x$. Sejam $\left\{E_{1}, E_{2}, \ldots, E_{n}\right\}$ em $\mathbb{R}_{2}^{n+4}$ campos tangentes a $Y$ consistindo de uma base ortonormal na métrica $g$ com base dual $\left\{\omega_{1}, \omega_{2}, \ldots, \omega_{n}\right\}$.

São satisfeitas as seguintes relações de ortogonalidade na métrica (5)

$$
\begin{aligned}
& \left\langle\Delta Y, E_{i}(Y)\right\rangle=0,\langle Y, \Delta Y\rangle=-n,\left\langle E_{i}(Y), E_{j}(Y)\right\rangle=\delta_{i j}, \\
& \langle Y, Y\rangle=\left\langle Y, E_{i}(Y)\right\rangle=0,\langle Y, \eta\rangle=\langle Y, P\rangle=0,\langle Y, N\rangle=-1, \\
& \langle N, N\rangle=0,\langle N, \eta\rangle=\langle N, P\rangle=\left\langle N, E_{i}(Y)\right\rangle=0, \\
& \langle\eta, \eta\rangle=\langle P, P\rangle=\left\langle\eta, E_{i}(Y)\right\rangle=0,\langle\eta, P\rangle=-1 .
\end{aligned}
$$

Então, temos a seguinte decomposição ortogonal

$$
\mathbb{R}_{2}^{n+4}=\operatorname{ger}\{Y, N\} \oplus \operatorname{ger}\left\{E_{1}(Y), E_{2}(Y), \ldots, E_{n}(Y)\right\} \oplus\{\eta, P\} .
$$

Chamamos $\left\{Y, N, E_{1}(Y), E_{2}(Y), \ldots, E_{n}(Y), \eta, P\right\}$ de referencial móvel de Laguerre em $\mathbb{R}_{2}^{n+4}$ de $x$. As equações de estrutura para este referencial são dadas por

$$
\begin{aligned}
& E_{i}(N)=\sum_{j} L_{i j} E_{j}(Y)+C_{i} P, \\
& E_{j}\left(E_{i}(Y)\right)=L_{i j} Y+\delta_{i j} N+\sum_{k} \Gamma_{i j}^{k} E_{k}(Y)+B_{i j} P, \\
& E_{i}(\eta)=-C_{i} Y+\sum_{j} B_{i j} E_{j}(Y),
\end{aligned}
$$

onde $C_{i}, L_{i j}=L_{j i}, B_{i j}=B_{j i}, \Gamma_{i j}^{k}$ são funções diferenciáveis definidas em $M^{n}$. Dessas equações temos os seguintes invariantes de Laguerre:

Definition 1. Definimos os invariantes $\mathbb{B}=\sum_{i j} B_{i j} \omega_{i} \otimes \omega_{j}, \mathbb{L}=\sum_{i j} L_{i j} \omega_{i} \otimes \omega_{j} e$ $\mathbb{C}=\sum_{i} C_{i} \omega_{i}$ denominados tensor segunda forma fundamental de Laguerre, tensor de Laguerre e forma de Laguerre, respectivamente.

Além dos invariantes básicos de Laguerre, a saber, métrica de Laguerre $g$, segunda 
forma fundamental de Laguerre $\mathbb{B}$, forma de Laguerre $\mathbb{C}$ e tensor de Laguerre $\mathbb{L}$, estudados pelos autores Li e Wang [6] para hipersuperfícies de Laguerre em $\mathbb{R}^{n+1}$, outros invariantes de Laguerre importantes são as curvaturas principais de Laguerre e as curvaturas de Laguerre.

Definição 6. Seja $x: M^{n} \rightarrow \mathbb{R}^{n+1}$ uma hipersuperfície orientável sem pontos umbílicos e com curvaturas principais não nulas. Denotamos os autovalores do operador da segunda forma fundamental de Laguerre $\mathbb{B}$ por curvaturas principais de Laguerre de $x$ dadas por

$$
b_{i}=\rho^{-1}\left(r-r_{i}\right)
$$

onde $r_{i}=\frac{1}{k_{i}}$ é o raio de curvatura, $r=\frac{r_{1}+\ldots+r_{n}}{n}$ é o raio de curvatura média de $x$ $e \rho=\sqrt{\sum_{i}\left(r-r_{i}\right)^{2}}$.

Definimos as curvaturas de Laguerre por:

Definição 7. Seja $x: M^{n} \rightarrow \mathbb{R}^{n+1}$ uma hipersuperfície orientável sem pontos umbílicos e com curvaturas principais não nulas. Para quaisquer três curvaturas principais $k_{i}, k_{j}$ e $k_{l}$, distintas, definimos

$$
\mathcal{L}^{i j l}=\frac{\left(k_{i}-k_{j}\right) k_{l}}{\left(k_{l}-k_{j}\right) k_{i}}
$$

como sendo a curvatura de Laguerre de $x$.

\section{Hipersuperfícies isoparamétricas de Laguerre em $\mathbb{R}^{n+1}$}

Considerando $x: M^{n} \rightarrow \mathbb{R}^{n+1}$ uma hipersuperfície com curvaturas principais que não se anulam e sem pontos umbílicos, sendo $g=\rho^{2} d \xi . d \xi$ a métrica de Laguerre onde $\xi: M^{n} \rightarrow$ $\mathbb{S}^{n}$ é o campo normal unitário de $x$ e $\rho=\sqrt{\sum_{i}\left(r-r_{i}\right)^{2}}$, introduzimos os conceitos de hipersuperfícies isoparamétricas de Laguerre e hipersuperfícies de Dupin.

Definição 8. Uma hipersuperfície $x: M^{n} \rightarrow \mathbb{R}^{n+1}$ é Dupin se suas curvaturas principais forem constantes ao longo de suas correspondentes linhas de curvatura, isto é, $e_{i}\left(k_{i}\right)=0$, onde $k_{i}$ é curvatura principal de $x$ e $e_{i}, i=1, \ldots, n$ é base canônica. Uma hipersuperfície de Dupin $x$ é dita própria se o número $r$ de curvaturas principais distintas for constante.

Definimos uma hipersuperfície isoparamétrica de Laguerre como sendo:

Definição 9. Seja $x: M^{n} \rightarrow \mathbb{R}^{n+1}$ uma hipersuperfície sem pontos umbílicos e com curvaturas principais que não se anulam. Dizemos que $x$ é uma hipersuperfície isoparamétrica de Laguerre se a forma de Laguerre $\mathbb{C}$ é nula e as curvaturas principais de Laguerre, dadas em (11), forem todas constantes. 
Definição 10. Uma hipersuperfície isoparamétrica de Laguerre $x: M^{n} \rightarrow \mathbb{R}^{n+1}$ é dita hipersuperfície isoparamétrica de Laguerre plana se

$$
R_{i j k l}=0, \quad \text { para todos } 1 \leq i, j, k, l \leq n,
$$

onde $R_{i j k l}$ é o tensor de curvatura de $g$.

No que segue, apresentamos exemplos de hipersuperfícies isoparamétricas de Laguerre, a saber, a cíclide de Dupin e $x: \mathbb{R}^{n} \rightarrow \mathbb{R}_{0}^{n+1}$ uma hipersuperfície orientada tipo-espaço em $\mathbb{R}_{0}^{n+1}$. Além disso, as hipersuperfícies descritas nos exemplos a seguir são também hipersuperfícies com segunda forma fundamental de Laguerre paralela. Uma hipersuperfície $x: M^{n} \rightarrow \mathbb{R}^{n+1}$ sem pontos umbílicos e com curvaturas principais que não se anulam possui segunda forma fundamental de Laguerre paralela se $\nabla \mathbb{B}=0$, onde $\nabla$ é a derivada covariante em relação à métrica $g$ e $\mathbb{B}$ é a segunda forma fundamental de Laguerre.

Exemplo 1. (Cíclide de Dupin) Para qualquer inteiro $m$ com $1 \leq m \leq n-1$ denotamos por

$$
\mathbb{H}^{n-m}=\left\{(v, w) \in \mathbb{R}^{n-m+1} / v \cdot v-w^{2}=-1, w>0\right\}
$$

o espaço hiperbólico imerso no espaço de Minkowski $\mathbb{R}_{1}^{n-m+1}$. Definimos $x: \mathbb{S}^{m} \times$ $\mathbb{H}^{n-m} \rightarrow \mathbb{R}^{n+1}$ por

$$
x(u, v, w)=\left(\frac{u}{w}(1+w), \frac{v}{w}\right), u \in \mathbb{S}^{m},(v, w) \in \mathbb{H}^{n-m} .
$$

A seguir apresentamos o exemplo de hipersuperfícies isoparamétricas de Laguerre planas dado por Li e Wang [7].

Exemplo 2. (Hipersuperfícies isoparamétricas de Laguerre planas) Para quaisquer intei$\operatorname{ros} m_{1}, m_{2}, \ldots, m_{s}, \operatorname{com} m_{1}+m_{2}+\cdots+m_{s}=n(s \geq 2)$ e constantes distintas não nulas $\lambda_{1}, \lambda_{2}, \ldots, \lambda_{s}$, definimos $x: \mathbb{R}^{n} \rightarrow \mathbb{R}_{0}^{n+1}$ uma hipersuperfície orientada tipo-espaço em $\mathbb{R}_{0}^{n+1}$ dada por

$x=\left(\frac{\lambda_{1}\left|u_{1}\right|^{2}+\lambda_{2}\left|u_{2}\right|^{2}+\cdots+\lambda_{s}\left|u_{s}\right|^{2}}{2}, u_{1}, u_{2}, \ldots, u_{s}, \frac{\lambda_{1}\left|u_{1}\right|^{2}+\lambda_{2}\left|u_{2}\right|^{2}+\cdots+\lambda_{s}\left|u_{s}\right|^{2}}{2}\right)$,

onde $\left(u_{1}, u_{2}, \ldots, u_{s}\right) \in \mathbb{R}^{m_{1}} \times \mathbb{R}^{m_{2}} \times \cdots \times \mathbb{R}^{m_{s}}=\mathbb{R}^{n},\left|u_{i}\right|^{2}=u_{i} \cdot u_{i}, i=1, \ldots, s$. Considerando a imersão de Laguerre $\tau: U \mathbb{R}_{0}^{n+1} \rightarrow U \mathbb{R}^{n+1}$, definida em Li e Wang [6], obtemos a hipersuperfície isoparamétrica de Laguerre associada $x^{\prime}: \mathbb{R}^{n} \rightarrow \mathbb{R}^{n+1}$, dada por

$$
x^{\prime}=\left(0, u_{1}, \ldots, u_{s}\right)+\frac{\sum_{i=1}^{s} \lambda_{i}\left|u_{i}\right|^{2}}{\sum_{i=1}^{s} \lambda_{i}^{2}\left|u_{i}\right|^{2}+1}\left(1,-\lambda_{1} u_{1}, \ldots,-\lambda_{s} u_{s}\right) .
$$


O resultado a seguir, obtido por Li e Wang [7], fornece uma classificação das hipersuperfícies de Dupin em $\mathbb{R}^{n+1}$ com $r$ curvaturas principais distintas $(r \geq 3)$ não nulas e curvaturas de Laguerre constantes.

Teorema 2. Seja $M^{n}$ uma hipersuperfície de Dupin em $\mathbb{R}^{n+1}$ com r curvaturas principais distintas $(r \geq 3)$ e não nulas. Então, $M^{n}$ é Laguerre equivalente a uma hipersuperfície isoparamétrica de Laguerre plana em $\mathbb{R}^{n+1}$ se, e somente se, as curvaturas de Laguerre são todas constantes.

Um passo importante na demonstração do Teorema 2 é a utilização de um resultado, obtido por Li, Li e Wang em [8], que classifica as hipersuperfícies com segunda forma fundamental de Laguerre paralela em termos das hipersuperfícies dadas no Exemplo 1]e no Exemplo2, Mais precisamente, o seguinte teorema:

Teorema 3. Seja $x: M^{n} \rightarrow \mathbb{R}^{n+1}$ uma hipersuperfície orientável sem pontos umbílicos com curvaturas principais que não se anulam. Se sua segunda forma fundamental de Laguerre $\mathbb{B}$ é paralela então $x$ é Laguerre equivalente a um subconjunto de umas das seguintes hipersuperfícies:

1. A cíclide de Dupin $x: \mathbb{S}^{k} \times \mathbb{H}^{n-k} \rightarrow \mathbb{R}^{n+1}$ dada por (13);

2. A hipersuperfície isoparamétrica de Laguerre $x: \mathbb{R}^{n} \rightarrow \mathbb{R}^{n+1}$ dada por (14).

O Teorema 3 é responsável por dizer de fato quem é a classe de hipersuperfícies isoparamétricas de Laguerre planas descrita no Teorema 2 ,

\section{Hipersuperfícies de Dupin em $\mathbb{R}^{n+1}$ com curvatura de Laguerre constante}

Em um contexto um pouco diferente do até então estudado, considerando uma hipersuperfície de Dupin própria $M^{n} \subset \mathbb{R}^{n+1}$ que admite um sistema de coordenadas principais e $n$ curvaturas principais distintas e não nulas, Cezana e Tenenblat [2] apresentaram uma caracterização para que as curvaturas de Laguerre sejam constantes em termos dos símbolos de Christoffel, de seus raios de curvatura e de sua primeira forma fundamental.

O principal teorema utilizado na demonstração do resultado de classificação, obtido por Cezana e Tenenblat [2], fornece uma caracterização das hipersuperfícies de Dupin em $\mathbb{R}^{n+1}, n \geq 3$, com todas as curvaturas principais distintas e curvaturas de Laguerre constantes, que admite uma parametrização por linhas de curvatura, em termos de seus raios de curvatura e de sua primeira forma fundamental. 
Teorema 4. Seja $x: U \subset \mathbb{R}^{n} \rightarrow \mathbb{R}^{n+1}$, com $n \geq 3$, uma hipersuperfície de Dupin própria, parametrizada por linhas de curvaturas $x\left(u_{1}, u_{2}, \ldots, u_{n}\right)$, com $n$ curvaturas principais $k_{i}, 1 \leq i \leq n$ distintas e não nulas e curvaturas de Laguerre constantes. Então, existem funções diferenciáveis $F_{i}\left(u_{i}\right), i=1, \ldots, n$ com $F_{i}^{\prime} \neq 0$ e $n$ constantes $\tilde{c}$, $\mathcal{C} \neq 0, D$ e $D_{s}, s \geq 4$, quando $n \geq 3$ satisfazendo

$$
D, D_{s} \in \mathbb{R}-\{0,1\}, D_{s} \neq \frac{1}{(1-D)}, D_{s} \neq D_{t}, \text { para } s \neq t, s, t \geq 4,
$$

tal que, a menos de reordenação dos índices, os raios de curvatura $r_{i}=\frac{1}{k_{i}}$ são dados por

$$
\begin{aligned}
& r_{1}=\frac{D}{4 \mathcal{C}(D-1)}\left[-F_{2}^{2}-\left(\frac{D-1}{D}\right) F_{3}^{2}+\sum_{s \geq 4} \frac{1}{\left(D_{s}-1\right)} F_{s}^{2}\right]-\mathcal{C}+\tilde{c} \\
& r_{2}=\frac{D}{4 \mathcal{C}(D-1)}\left[F_{1}^{2}-\left(\frac{D-1}{D^{2}}\right) F_{3}^{2}+\sum_{s \geq 4} \frac{D_{s}}{\left(D_{s}-1\right)^{2}} F_{s}^{2}\right]-\frac{\mathcal{C}}{D}+\tilde{c} \\
& r_{3}=\frac{D}{4 \mathcal{C}(D-1)^{2}}\left[D F_{1}^{2}+F_{2}^{2}+\sum_{s \geq 4} \frac{D D_{s}-D_{s}+1}{\left(D_{s}-1\right)^{2}} F_{s}^{2}\right]+\tilde{c} \\
& r_{s}=D_{s} r_{1}-\left(D_{s}-1\right) r_{2}, \quad \text { para } s \geq 4,
\end{aligned}
$$

onde $D=\mathcal{L}^{132}, D_{s}=\mathcal{L}^{s 21}$ e a métrica diagonal dada por

$$
\sqrt{g_{11}}=\frac{-F_{1}^{\prime} r_{1}}{r_{2}-r_{1}} e \sqrt{g_{j j}}=\frac{F_{j}^{\prime} r_{j}}{r_{j}-r_{1}}, \quad j \geq 2 .
$$

Reciprocamente, dadas funções diferenciáveis $F_{i}\left(u_{i}\right), i=1, \ldots, n, \geq 3$, com $F_{i}^{\prime} \neq 0$ e $n$ constantes $\tilde{c}, \mathcal{C} \neq 0, D$ e $D_{s}, s \geq 4$ satisfazendo (15), a menos de movimento rígido, existe uma única hipersuperfície de Dupin em $\mathbb{R}^{n+1}$ com curvaturas de Laguerre constantes $D=\mathcal{L}^{132}, D_{s}=\mathcal{L}^{s 21}$, parametrizada por linhas de curvatura, cujos raios de curvatura e a métrica são dadas por (16) e (17), respectivamente.

Assim, a classificação proposta por Cezana e Tenenblat [2] consiste em uma verificação de que a família de hipersuperfícies de Dupin em $\mathbb{R}^{n+1}$ descrita em (1) satisfazem as condições do Teorema 4. Mais precisamente, Cezana e Tenenblat [2] obtiveram o seguinte resultado:

Teorema 5. Qualquer hipersuperfície de Dupin própria de $\mathbb{R}^{n+1}$, com n curvaturas principais distintas e não nulas, que tem sistema de coordenadas principais e curvaturas de Laguerre constantes, é dada por (1), a menos de movimento rígido e a menos de mudança de cada variável independente separadamente. 
i) As constantes associadas a esta hipersuperfície são então $(n-2)$ curvaturas de Laguerre

$$
D=\mathcal{L}^{132}=\frac{\left(b_{1}-b_{3}\right) b_{2}}{\left(b_{2}-b_{3}\right) b_{1}}, D_{s}=\mathcal{L}^{s 21}=\frac{\left(b_{s}-b_{2}\right) b_{1}}{\left(b_{1}-b_{2}\right) b_{s}}, s=4 \ldots n
$$

e duas constantes

$$
\mathcal{C}=\frac{b_{1}-b_{3}}{2 b_{1} b_{3}}, \tilde{c}=\frac{1}{2 b_{3}}-\gamma
$$

ii) Reciprocamente, são dadas $n$ constantes $\tilde{c}, \mathcal{C} \neq 0, D$ e $D_{s}, s \geq 4$ satisfazendo

$$
D, D_{s} \in \mathbb{R}-\{0,1\}, D_{s} \neq \frac{1}{(1-D)}, D_{s} \neq D_{t}, \text { para } s \neq t, s, t \geq 4
$$

seja $X(0)=(0, \ldots, 0, \gamma) \in \mathbb{R}^{n+1}$ tal que

$$
(\gamma+\tilde{c}) \notin\left[0, \mathcal{C}, \frac{\mathcal{C}}{D}, \frac{\left[(D-1) D_{s}+1\right] \mathcal{C}}{D}\right] .
$$

Seja

$$
\begin{aligned}
& b_{1}=\frac{1}{2(\gamma+\tilde{c}+\mathcal{C})}, \quad b_{2}=\frac{D}{2[D(\gamma+\tilde{c})-\mathcal{C}]} \\
& b_{3}=\frac{1}{2(\gamma+\tilde{c})}, \quad b_{s}=\frac{D}{2\left\{D(\gamma+\tilde{c})-\mathcal{C}\left[D_{s}(D-1)+1\right]\right\}} .
\end{aligned}
$$

Então, a família a 2 parâmetros da hipersuperfície definida por (1) tem curvaturas de Laguerre constantes $D$ e $D_{s}$.

\section{Conclusão}

Usando técnicas distintas, os autores Li e Wang [7] e Cezana e Tenenblat [2] encontraram exemplos de hipersuperfícies de Dupin em $\mathbb{R}^{n+1}$ com curvaturas de Laguerre constantes. Li e Wang [7], em 2015, mostraram (Teorema 2) que uma hipersuperfície de Dupin, com $r$ curvaturas principais distintas ( $r \geq 3$ ) e não nulas, e curvaturas de Laguerre constantes é Laguerre equivalente a isoparamétrica de Laguerre plana dada por (14) no Exemplo2.

Em 2017, Cezana e Tenenblat [2] mostraram um resultado mais geral (Teorema 5) que afirma que qualquer hipersuperfície de Dupin em $\mathbb{R}^{n+1}$ com $n$ curvaturas principais distintas e não nulas, que admite um sistema de coordenadas principal, com curvaturas de Laguerre constantes é dada pela hipersuperfície (1). Observamos que a classe de hipersuperfícies descrita em (14), no Exemplo 2, é um caso particular da família dada em (1) quando $\gamma=0$. 


\section{Referências}

[1] CECIL, T. E.: Lie Sphere Geometry: with applications to submanifolds, Springer Verlag, Berlin Heidelberg, New York, 1992.

[2] CEZANA, M. J.; TENENBLAT, K.: Dupin hypersurfaces with constant Laguerre curvatures, Manuscripta Math., v.154, p.169-184, 2017.

[3] CORRO, A. V.; FERREIRA, W.; TENENBLAT, K.: On Ribaucour transformations for hypersurfaces, Mat. Contemp., v.17, p.137-160, 1999.

[4] CAIXETA, F. V.; RODRIGUES, L. A.: Geometria de Laguerre e hipersuperfícies de Dupin com curvatura de Laguerre constante em $\mathbb{R}^{n+1}$, p.122, Dissertação de Mestrado, Departamento de Matemática, Universidade de Brasília, Brasília-DF, 2019.

[5] FERRO, M. L.; RODRIGUES, L. A.; TENENBLAT, K.: On Dupin Hypersurfaces in $\mathbb{R}^{5}$ parametrized by lines of curvature, Results. Math, v.70, p.499-531, 2016.

[6] LI, T. Z.; WANG, C. P.: Laguerre geometry of hypersurfaces en $\mathbb{R}^{n}$, Manuscripta Math., v.122, p. 73-95, 2007.

[7] LI, T. Z.; QING, J.; WANG, C. P.: Möbius and Laguerre geometry of Dupin hypersurface, UC Santa Cruz Previously Published Works, v.122, 2015.

[8] LI, T. Z.; LI, H.; WANG, C. P.: Classification of hypersurfaces with parallel Laguerre second fundamental form in $\mathbb{R}^{n}$, Differential Geom. Appl., v.28, p.148$157,2010$.

[9] LI, T. Z.; SUN, H. F.: Laguerre isoparametric hypersurfaces in $\mathbb{R}^{4}$, Acta Math. Sinica, English Series, v.28, p.1179-1186, 2011.

[10] PINKALL, U.: Dupin hypersurfaces, Math. Ann., v.270, p.427-440, 1985.

[11] RIVEROS, C. M. C.; RODRIGUES, L. A.; TENENBLAT, K.: On Dupin hypersurfaces with constant Möbius curvature, Pacific Journal of Mathematic, v.236, n.1, p.89-103, 2008.

】

Submetido em 31 mar. 2020

Aceito em 10 jun. 2020 